\title{
Pédagogie médicale : un facteur de rassemblement et de solidarité dans la francophonie médicale
}

\begin{abstract}
a création d'une revue internationale francophone de pédagogie médicale ne relève pas du hasard. Elle refletel'importance acquise par la pédagogie des sciences de la santé et répond à un besoin de diffusi on et de partage des expériences entreprises dans un nombrede plus en plus grand de facultés de médecine du monde francophone.
\end{abstract}

La pédagogie médicale telle qu'elle se présente actuellement et se profile à l'avenir a révolutionné I'enseignement de la médeane et suscite l'enthousiasme par les valeurs humanistes d'éducation et de développement qu'elle soustend. Cette pédagogie nouvelle est personnalisée, elle et centrée sur l'apprenant, elle suppose la connais sance de celui qu'on veut accompagner, le désir qui l'anime, ses possibilités, ses interrogations: "Toute leçon doit être une réponse » a-t-on dit à justetitre. $M$ ais elle est aussi communautaire; ell e fait vivre et travailler lesétudiants ensemble, en leur apprenant à sexprimer, à sécouter, à se respecter, à se « socialiser » à traversla dynamique de groupe où la confrontation des personnes peut être régulée par l'exigence de verité Cette pédagogie est active; elle met l'étudiant en situation d'apprendre et d'agir par lui-même dans une situation où la véritése découvre et se construit et n'est pas relative au statut de celui qui l'énonce. Cette pédagogie sadresse enfin à une liberté qu'elle vise à épanouir loin de toute «colonisation inté rieure».

La pédagogie médicale et ains devenue sans nul doute une di scipline à part entière avec ses sciences fondamentales, ses applications « académiques", ses centres de recherche, ses vedettes qui, pour certaines déjà, bientôt pour d'autres, sont ou seront des figures de légende. Comme toute discipline en évolution, elle nécessite toutefois confrontation et échanges Plus nous avançons en effet, plus les sá ences fondamental es explorant les mécani smes de fonctionnement de la mémoire I'organisation des connaissances, se révètent complexes et encore à explorer. Plus nous avançons, plus nous devons adapter nos pratiques d'enseignement aux nouvelles acquistions et aux moyens de plus en plus performants dont nous disposons. C'est ains que l'intrusion massive des nouvelles technologieset leur montrée en puissance créent un monde véritablement nouveau où les règles du jeu social et interpersonne vont radical ement dhanger. $N$ ous ne mesurons pas toujours l'étendue de notre ignorance. Dans un texte intitulé « Q u'et-ce qu'on ne sait pas», le philosophe Paul Ricoeur dit ceci : «Le savoir augmenteet on ne peut pas dire del'ignorancequ'el le diminue II sopère plutôt une complexification de ce que l'on croyait simple »

Les conférences, séminaires et colloques qui se sont succédés dernièrement dans le monde francophone reflètent l'intérêt portéà la pédagogie et à sa pérennitédésormaisbien établie II devenait urgent d'envisager la création d'une Revue pour permettre une large diffusion des problématiques, des méthodologiesains que de la miseen place d'un forum destinéà favoriser le partage des expérienceset des résultats. C'est maintenant chose faite. La Revue qui paraissait encore une "utopie » il n'y a pas longtemps, es désormais une réalité. Nous la devons à la compétence et à la ténacité de quelques pionniers : Bernard CHARLIN, Jean-François DENEF et Raymond COLIN .

Cette revue sera un facteur de rassemblement et de solidarité dans la francophonie médicale qui sest érigée au fil desans en un véritable réseau d'estime et d'amitié grâce en particulier à l'action de la Conférence Internationale des $D$ oyens des Facultés de M édecine d'Expression Française (CID M EF) et de son Préident fondateur AndréG OU AZÉ qui a su grouper et faire travailler ensemble des hommes d'horizons divers pour une coopération médicale universitaire dans un esprit de service, de libertéet de multilatéralité. Face à une mondialisation qui par certains aspects cherche à imposer un mode de vie et de réflexion global unique, cette Revue Internationale Francophone permettra sans nul doute de "promouvoir et féconder les cultures propres à chaque pays parce qu'elle retera ouverte 
aux différences, et consolidera, tout en l'ouvrant sur l'altérité, l'identité culturelle de ceux qui ont adopté le français comme langue de culture ». (Sélim ABOU : Les identités en quetion)

L'ambition de toute pédagogie vise l'épanouissement d'une personne en fonction de ses aptitudes et de ses aspirations pour qu'au service des autres elle puisse devenir le membre actif d'une profession ou d'une société. Puisse cette Revue contribuer à aider les ensegnants de la santé à devenir des éducateurs, " car il n'est rien de s beau et légitime que de bien fairel'H omme » (M ontaigne).

Pr PierreFARAH

D oyen de la Faculté de médecine de I'U niversité Saint-J oseph, Beyrouth Président de la CIDMEF 